\title{
Long-timescale simulations of diffusion in molecular solids
}

\author{
L.J. Karssemeijer, ${ }^{a}$ A. Pedersen, ${ }^{b}$ H. Jónsson, ${ }^{b}$ and H.M. Cuppen ${ }^{* a}$
}

\author{
Received Xth XXXXXXXXXX 20XX, Accepted Xth XXXXXXXXX 20XX \\ First published on the web Xth $X X X X X X X X X X 200 X$ \\ DOI: $10.1039 / b 000000 x$
}

Kinetic processes play a crucial role in the formation and evolution of molecular layers. In this perspective we argue that adaptive kinetic Monte Carlo is a powerful simulation technique for determining key kinetic processes in molecular solids. The applicability of the method is demonstrated by simulating the diffusion of a $\mathrm{CO}$ admolecule on a water ice surface, which is an important process for the formation of organic compounds on interstellar dust grains. $\mathrm{CO}$ diffusion is found to follow Arrhenius behavior and the corresponding effective activation energy for diffusion is determined to be $50 \pm 1 \mathrm{meV}$. A coarse graining algorithm is applied which greatly enhances the efficiency of the simulations at low temperatures, down to $10 \mathrm{~K}$, without altering the underlying physical processes. Eventually, we argue that a combination of both on- and off-lattice kinetic Monte Carlo techniques is a good way for simulating large-scale processes in molecular solids over long time spans.

\section{Introduction}

\subsection{Molecular solids}

Layers of solid molecular material, either in amorphous, crystalline or microcrystalline form, are not as rigid as one might intuitively expect. Since the structuring and mobility in these layers is dominated by weak interactions, bonds can easily be broken and reformed. As such, molecular layers can play an important role as the interface between the gas phase or solution and the bulk of the solid, probably facilitating the onset of phase separations in mixed layers and structural changes. An example of this effect is shown in Fig. 1. The top panel shows lattice-gas Monte Carlo simulations of the segregation of a binary solid mixture using a toy model. Comparison to the segregation of a $\mathrm{H}_{2} \mathrm{O}: \mathrm{CO}_{2}=1: 1$ mixture at $55 \mathrm{~K}$ (bottom panel) shows that a combination of bulk (interchanging molecules) and surface (surface diffusion) processes needs to be included to reproduce the experimentally observed trends ${ }^{1}$.

Mobility in molecular solids can further promote chemical reactions by allowing reactants to meet. Restructuring will occur once the product has formed, where the excess energy can be applied in accommodating the local environment for the newly-formed molecule. These restructuring processes are central to many fields and environments, e.g., ice layers on dust particles in the atmosphere and enantiomer separation ${ }^{2}$. In all cases, understanding the restructuring mechanism will shed light on the nature of the entire system ${ }^{3}$. In atmospheric chemistry it is well recognized that small aerosol particles and

a Radboud University Nijmegen, Institute for Molecules and Materials, Heyendaalseweg 135, 6525 AJ Nijmegen, The Netherlands. E-mail: h.cuppen@science.ru.nl

${ }^{b}$ Faculty of Science and Science Institute, University of Iceland, 107 Reykjavík, Iceland ice crystals are involved as catalysts in the conversion of many molecules. Understanding these reactions is crucial to gain insight into greenhouse-gas chemistry ${ }^{4}$.

In the present paper, we focus specifically on the formation and evolution of interstellar ices, which is important for questions dealing with star and planet formation and ultimately with the origin of life, specifically concerning the delivery of molecules - like $\mathrm{H}_{2} \mathrm{O}, \mathrm{CO}_{2}$ and organic molecules - to habitable planets. Molecules that are present in ices on dust particles can become trapped during accretion from dust to planet, or may be transported by comets to existing planets. In either case, interstellar ices are at the origin of the mechanism ${ }^{5,6}$. It is the physics and chemistry of interstellar ices which determines the particular molecular species that will be trapped in ices during star and planet formation and can therefore be delivered to planets.

\subsection{Interstellar ices}

The interstellar medium is far from empty, rather it contains large molecular clouds consisting of dust grains and gas. It is in these clouds that new stars form - possibly with habitable planets - with molecules playing a crucial role ${ }^{7,8}$. Some of the ice species accrete from the gas phase, but as astrochemists have only just started to realize - many of the simple important molecules such as $\mathrm{H}_{2}$ and $\mathrm{H}_{2} \mathrm{O}$ but also the more complex organic molecules are not predominantly formed in the gas phase, but rather on the grain surfaces themselves. Generally speaking, gas phase chemistry leads to more unsaturated molecules as compared to grain surface chemistry ${ }^{9}$. The main components of the ice mantle are $\mathrm{H}_{2} \mathrm{O}, \mathrm{CO}$ and $\mathrm{CO}_{2}$, but more complex molecules may also be present ${ }^{10,11}$. A schematic representation of an interstellar

This journal is @ The Royal Society of Chemistry [year]

Journal Name, 2010, [vol], 1-10 |1 


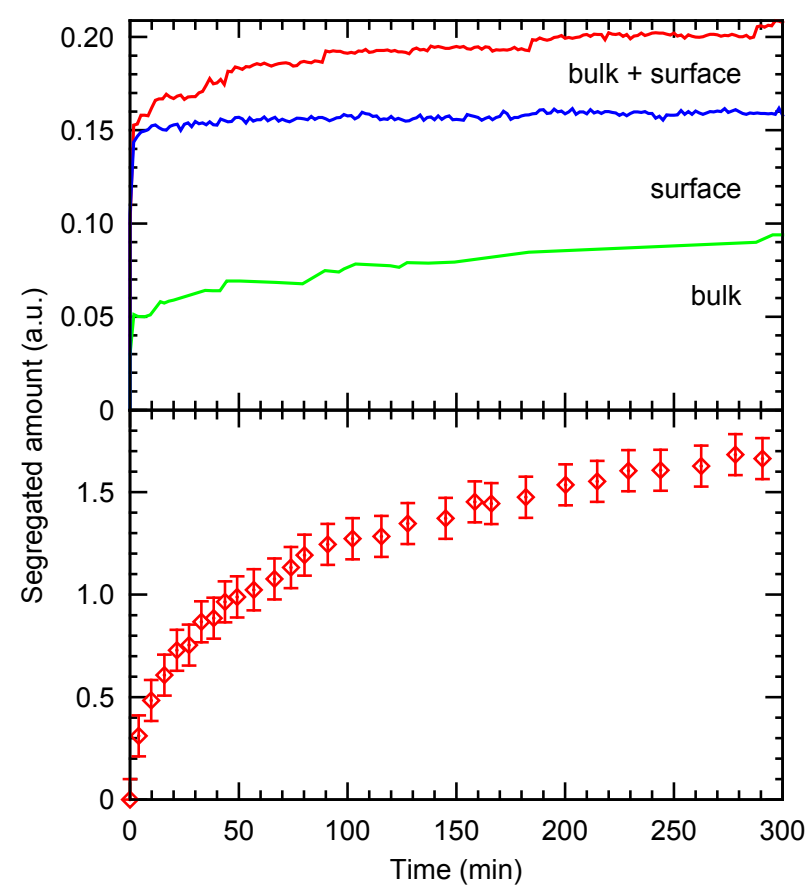

Fig. 1 Monte Carlo simulations of ice segregation using a toy model (top panel). A combination of bulk (interchanging molecules) and surface (surface diffusion) processes needs to be included to reproduce the experimentally observed segregation in a $\mathrm{H}_{2} \mathrm{O}: \mathrm{CO}_{2} 1: 1$ mixture at $55 \mathrm{~K}$ (bottom panel). Results published in Ref. ${ }^{1}$.

dust grain is shown in Fig. 2.

Most of our current knowledge on interstellar ices springs from a combination of infrared observations and laboratory experiments. The line profiles of infrared observations of ices give information in terms of mixtures and structure. Different local molecular environments lead to line broadening and/or peak shifting. In a recent article we showed that $\mathrm{CO}$ is most likely mixed with $\mathrm{CH}_{3} \mathrm{OH}$ and not with $\mathrm{H}_{2} \mathrm{O}$ in interstellar ices near Young Stellar Objects ${ }^{12}$. This information was obtained by comparing laboratory and observational infrared spectra and the findings have implications for the possible environments in which $\mathrm{H}_{2} \mathrm{O}$ and $\mathrm{CH}_{3} \mathrm{OH}$ are formed in space. In a similar way, segregation of $\mathrm{CO}_{2}$ from $\mathrm{H}_{2} \mathrm{O}$ has been observed near young stars ${ }^{13}$. This observed segregation could be used as a tracer for environment temperature or age of the object once we understand the microscopic phenomena of the process and we can make predictions about the timescales and temperatures at which this occurs.

The realization that grain surface chemistry plays an important role in the interstellar medium has opened a new, mostly unexplored, research field. Questions that have been answered already for decades for gas phase chemistry are just recently being addressed for grain surface chemistry.

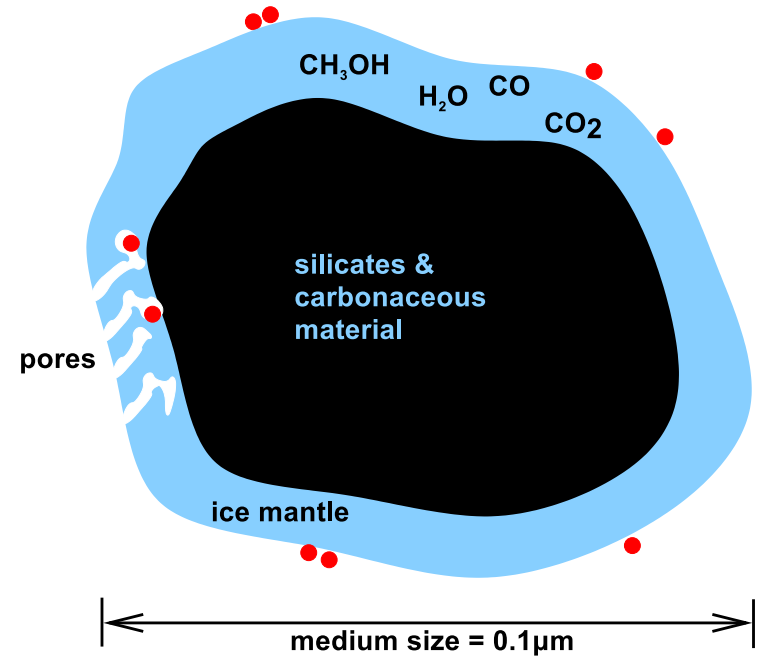

Fig. 2 Schematic representation of a dust grain with ice mantle. Adsorbents on the surface and in pores can react with each other.

Many modelers have begun to include grain-surface processes in their models to describe chemical evolution during, for instance, star formation ${ }^{7,14-16}$. The problem is, however, that many of the input parameters for surface chemistry are highly uncertain or simply unknown. Moreover, the correct formalism itself is debated ${ }^{17,18}$. Before we can make accurate predictions on the molecules that could form on these interstellar ices, we must gain an understanding of the processes that bring the reactants together in these icy layers.

Most of the current astrochemical gas-grain models use rate equation treatments to simulate grain chemistry ${ }^{8,19-21}$. One of the intrinsic characteristics of this mean-field method is that the actual structure of the ice and the exact location of all species are not included. Recent computational and experimental studies of the formation of $\mathrm{H}_{2} \mathrm{O}$ and $\mathrm{CH}_{3} \mathrm{OH}^{22,23}$ indisputably showed that the structure of the ice is central to the reactivities and lifetimes of molecules in the ice. Attempts are underway to increase the amount of detail in the current models, ${ }^{24}$; however, as long as we lack an understanding of the processes that control the formation of icy mantles, the introduction of more complex expressions will not necessarily lead to a more accurate description. Several studies ${ }^{18,23,25}$ have shown that as soon as one leaves the submonolayer regime, i.e., once ice layers start to build up, the chemistry becomes much more complex and less intuitive.

In the present paper we will move beyond rate equations and will show how state-of-the-art physico-chemical techniques can cover large timescales while treating the system with atomistic detail. 


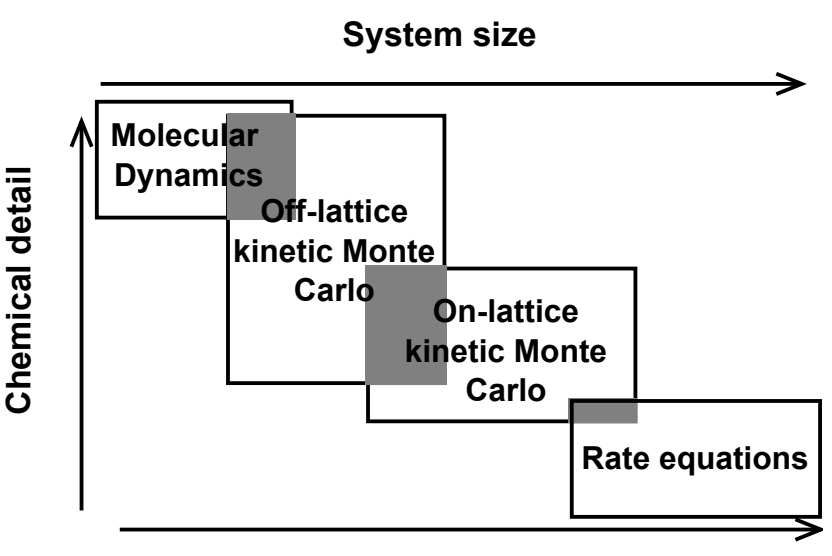

Time

Fig. 3 Overview of several different simulation methods and their applicability.

\subsection{Simulation methods}

The fundamental difficulty in modeling interstellar ices is that they are essentially rare-event systems, mainly due to the low temperatures. Molecular movement on picosecond timescales leads to larger structural changes which occur only after hours, or even years. This implies a timescale range of roughly 20 orders of magnitude, far beyond the reach of traditional molecular dynamics. We can however tackle this problem using a combination of Monte Carlo simulation techniques.

As Fig. 3 shows, kinetic Monte Carlo (KMC) techniques are able to cover a wide range of complexity and timescales. $\mathrm{KMC}$ is a specific type of Monte Carlo technique which differs from ordinary Metropolis Monte Carlo mainly by its ability to trace the passing of time. This does, however, require the use of transition rates instead of just probabilities for new configurations to be accepted. The time increment in an iteration in a $\mathrm{KMC}$ simulation is given by

$$
\Delta t=-\frac{\ln x}{\sum_{i} k_{i}},
$$

where $x \in(0,1]$ is a random number, $k_{i}$ is the transition rate of process $i$ and the sum runs over all processes which are accessible from the current state of the system. Because of this sum, all possible processes in a given cycle of the simulation need to be known before it can be executed. These processes are stored in the so-called table of events (TOE). In contrast, an iteration in the Metropolis Monte Carlo algorithm only involves the energy difference between a pair of configurations, without any reference to rates of transitions.

Two types of KMC are distinguished in Fig. 3: on-lattice and off-lattice KMC. A clear advantage of an on-lattice model is that one can work with a predefined TOE. Since the molecules are confined to a lattice, only a limited number of events are possible, for which the rates can be determined at the start of the simulation. For our purpose however, this is also a drawback. Since we want to simulate amorphous systems, making guesses about the possible events is far from straightforward and it is easy to miss important mechanisms. As we will show in Section 3, even well-defined crystalline systems can exhibit unexpected processes. Another disadvantage of having the molecules at fixed sites is that most molecular detail is lost and the use of realistic potentials is not straightforward; although this can be circumvented in crystalline molecular solids by calculating a crystal graph ${ }^{26}$.

The other type of KMC, off-lattice KMC, does not restrict the molecules to predefined lattice sites. This allows for realistic potentials to be used and gives access to the full molecular geometry. However, one cannot use a predefined TOE since it is impossible to determine all possible transitions that the system could experience throughout the simulation beforehand. A solution to this problem is provided in adaptive kinetic Monte Carlo (AKMC) ${ }^{27-29}$. In this off-lattice KMC technique, the TOE is constructed on the fly and only the processes leading out of the current state of the system are considered. Here, a state is defined as a local minimum on the potential energy surface (PES). This is another contrast with Metropolis Monte Carlo, where trial moves do not necessarily represent local minima on the PES. In AKMC, the processes in the TOE are determined by performing a large number of saddle point searches to construct a transition state dividing surface which separates the current state from its neighbors. These searches are carried out using the minimum mode following algorithm ${ }^{30}$. The particular implementation of AKMC we use in this paper, $\mathrm{EON}^{28,31}$, can distribute the searches over a large number of different, and possibly remote, machines to speed up the simulation. Using harmonic transition state theory ${ }^{32}$, the rates for crossing the transition states are calculated and these are then used to populate the TOE. Once the table is considered complete, a process is selected according to its relative rate using a random number and the time is advanced according to Eq. (1). The saddle point search procedure then starts over in the new state. If the system revisits a state, the TOE that has been constructed in a previous visit can be reused. In this way, the simulation becomes less CPU demanding when more states have been visited or when additional simulations, with different conditions (temperature, random seed), need to be carried out. The simulations can be accelerated further by applying a coarse graining method as is discussed in Section 3.2. A drawback of the AKMC method compared to on-lattice models is that it is computationally rather expensive (due to the saddle point searches) which limits the size of the systems it can treat.

In our approach, we will use both off-lattice AKMC and onlattice KMC to bridge the timescale gap between the picosecond individual molecular movements and the, much slower, 
timescale for restructuring effects. The AKMC method is particularly useful for simulating complex, rare-event systems, where the kinetic molecular processes are often non-intuitive. On-lattice KMC, on the other hand, has proved its worth when simulating large systems over long timescales ${ }^{18,25,33,34}$; but the TOE needs to be determined beforehand. Our strategy is therefore to use AKMC for determining which typical kind of processes form the crucial transition steps and then translate this data into an on-lattice model. This approach should be able to really mimic laboratory experiments accurately, covering hours of simulation time.

Since on-lattice KMC has been used for the simulation of mixed icy layers before, we will focus on the application of $\mathrm{AKMC}$ to molecular systems in the remainder of this paper.

\section{AKMC method for molecular systems}

Adaptive kinetic Monte Carlo has been demonstrated to work efficiently for atomic systems $27,35,36$. For the simulation of interstellar ices however, we need to progress to molecular solids. Here, the main complication arises due to the presence of both strong intramolecular forces and much weaker intermolecular forces. As a consequence, the PES becomes much complex than that of an atomic system. This makes it harder to locate saddle points using the minimum mode following algorithm since the lowest energy eigenvector will vary rapidly with only small variations in the atomic coordinates.

To demonstrate the suitability of the AKMC method for long timescale simulation of molecular solids, we apply it here to a system consisting of an ice $\mathrm{I}_{\mathrm{h}}(0001)$ substrate with a single $\mathrm{CO}$ admolecule. The paths generated for the $\mathrm{CO}$ molecule are used to compute surface diffusion constants at astronomically relevant temperatures reaching down to $10 \mathrm{~K}$. All simulations were performed using the EON ${ }^{28,31}$ software package.

\subsection{Potentials}

All interactions in the system are described by classical pair potentials. Molecules are treated as fully flexible, with intramolecular forcefields restraining bond lengths and angles. Since our system contains multiple $\mathrm{H}_{2} \mathrm{O}$, and a single $\mathrm{CO}$ molecule, we need two forcefields. One for $\mathrm{H}_{2} \mathrm{O}-\mathrm{H}_{2} \mathrm{O}$, and another for the $\mathrm{H}_{2} \mathrm{O}-\mathrm{CO}$ interactions.

Interactions between water molecules are described by the TIP4P/Ice potential ${ }^{37}$ which has been specifically fitted to reproduce properties of the solid phases of $\mathrm{H}_{2} \mathrm{O}$. Intramolecular interactions within the water molecules are treated by the quartic potential proposed by Carney et al. ${ }^{38}$ (CCL).

The $\mathrm{H}_{2} \mathrm{O}-\mathrm{CO}$ potential was fitted to $\mathrm{CCSD}(\mathrm{T}$ ) (aug-ccpVTZ, BSSE corrected) calculations of the two-molecule complex. For reasons of consistency and computational efficiency, the charges on the $\mathrm{H}_{2} \mathrm{O}$ molecules are identical to the TIP4P/Ice charges. CO molecules are modeled with three charged sites, one on each atom and a third one on the center of mass. The charges on the atoms depend on the bond length in the molecule through the relation:

$$
q_{i}=q_{i}^{0} \exp \left\{-\sigma_{i}\left(r_{\mathrm{CO}}-r_{\mathrm{CO}}^{0}\right)\right\} \quad(i=\mathrm{C}, \mathrm{O}),
$$

where $q_{i}^{0}$ is the charge in equilibrium configuration, $r_{\mathrm{CO}}$ is the instantaneous bond length and $r_{\mathrm{CO}}^{0}$ is the equilibrium bond length. The third charge is such that the molecule has zero charge. This model was proposed in order to reproduce the dipole and quadrupole moments from CI (aug-cc-pVTZ) calculations ${ }^{39}$. In addition to the electrostatics, a Buckingham potential is applied to each atomic intermolecular pair:

$$
U_{\text {Buckingham }}(r)=A \exp (-B r)-\frac{C}{r^{6}} .
$$

Intramolecular interactions within each $\mathrm{CO}$ molecule are described by a Morse potential:

$$
U_{\text {Morse }}\left(r_{\mathrm{CO}}\right)=D_{e}\left[1-\exp \left\{\gamma\left(r_{\mathrm{CO}}-r_{\mathrm{CO}}^{0}\right)\right\}\right]^{2} \text {. }
$$

Experimental values of $D_{e}$ and $r_{\mathrm{CO}}^{0}$, which agree to within 0.1\% with CCSD(T) (aug-cc-pVQZ) calculations, were used for this potential ${ }^{40-42}$ The parameter $\gamma$ was then fitted to reproduce the bond length dependence from the ab-initio calculations ${ }^{39}$. The values of all parameters of the $\mathrm{H}_{2} \mathrm{O}-\mathrm{CO}$ potential are listed in Table 1 .

All interactions are switched off between 9 and $10 \AA$ (center of mass distance between a pair of molecules) using the function $f(x)=(2 x-3) x^{2}+1$ with $x \in(0,1)$. Ewald summation is not applied for the calculation of long range electrostatics. One argument for this is that during the saddle point searches, significant motion of atoms is confined to a finite region around the admolecule, and changes in energy will therefore be dominated by short-range electrostatics. Furthermore, the restriction to (effective) pair potentials and the ignored polarizability is likely to have a more severe effect on the energy barriers than the less accurate treatment of the Coulombic interactions.

\subsection{Simulation details}

To initialize a saddle point search, the system is slightly displaced from its current configuration in a minimum of the PES. It is known for atomic systems that a Gaussian displacement of atoms in the region of interest yields the highest efficiency in finding the low-lying saddle points leading out of the initial basin in the PES ${ }^{43}$. In our simulations however, molecules are displaced instead of atoms. This is done by a translation of the molecule along the three Euclidean axes, followed by a rotation about each axis. Magnitudes of the 
Table 1 Parameters for the $\mathrm{H}_{2} \mathrm{O}-\mathrm{CO}$ potential

\begin{tabular}{llll}
\hline & \multicolumn{3}{l}{$\mathrm{H}_{2} \mathrm{O}-\mathrm{CO}$ Buckingham parameters } \\
Interaction & $\mathrm{A}(\mathrm{eV})$ & $\mathrm{B}\left(\AA^{-1}\right)$ & $\mathrm{C}\left(\mathrm{eV} \AA^{6}\right)$ \\
\hline $\mathrm{O}-\mathrm{C}$ & 427.9 & 2.725 & 70.38 \\
$\mathrm{O}-\mathrm{O}$ & $6.867 \times 10^{7}$ & 8.022 & 1.890 \\
$\mathrm{H}-\mathrm{C}$ & 667.2 & 4.506 & 1.942 \\
$\mathrm{H}-\mathrm{O}$ & 537.7 & 4.551 & $1.290 \times 10^{-3}$ \\
\hline \multicolumn{4}{c}{$\mathrm{CO}$ intramolecular parameters } \\
\hline$r_{\mathrm{CO}}^{0}$ & $1.128 \AA$ & $\sigma_{\mathrm{C}}$ \\
$q_{\mathrm{C}}^{0}$ & $-0.470 e$ & $\sigma_{\mathrm{O}}$ & $3.844 \AA^{-1}$ \\
$q_{\mathrm{O}}^{0}$ & $-0.615 e$ & $D_{e}$ & $11.23 \AA^{-1}$ \\
& \multicolumn{3}{c}{}
\end{tabular}

displacements and angles of rotation are drawn from Gaussian distributions with predefined standard deviation. This approach ensures that the internal geometry of the molecules remains unchanged.

The initial displacement starts with the selection of a molecule of interest: the admolecule. Next, a choice is made between a single molecule displacement (40\% probability) and a multiple-molecule displacement of all molecules which have at least one atom within a $4 \AA$ radius any atom of the admolecule ( $60 \%$ probability). In the case of a single molecule displacement, the standard deviation is $0.6 \AA$ for displacements and 0.5 radians for rotations. For multiple-molecule displacements, these standard deviations are $0.3 \AA$ and 0.4 radians, respectively.

The saddle point searches are conducted using the minimum mode following method ${ }^{30}$, using the Lanczos algorithm to estimate the lowest eigenvector of the Hessian ${ }^{44}$. Despite the presence of both intra- and intermolecular forces, tests show that the saddle point searches have a success rate of approximately $60 \%$ in finding a saddle point, which shows that the algorithm is rather robust and can be applied to molecular systems. The system is considered to be converged on a saddle point when the Hessian has one negative eigenvalue and the force on any atom is below $10^{-3} \mathrm{eV} / \AA$. Minima in the PES are converged to a stricter value of $10^{-4} \mathrm{eV} / \AA ̊$. Any processes with energy barriers more than $30 \mathrm{kT}$ above the lowest barrier from the state are not included in the TOE since these are unlikely to be chosen.

An advantage of KMC simulations is that, once the TOE is known for a specific temperature, it is simple to simulate other temperatures. Since the process barriers and prefactors are temperature independent, one can simply recalculate the rates for a new temperature using the harmonic transition state theory expression ${ }^{32}$ :

$$
k=v \exp \left(-E_{b} / k T\right),
$$

where $v$ is the process prefactor and $E_{b}$ the energy barrier. By performing an AKMC simulation at the highest temperature of interest first, it is then - with the already completed TOE — straightforward to perform additional runs at lower temperatures.

The ice $I_{h}$ substrate contains 360 water molecules arranged in three bilayers. The initial hydrogen bond network is generated using the method of Buch et al. ${ }^{45}$. It has a negligible dipole moment with periodic boundary conditions applied in all three dimensions. Next, all atomic coordinates are relaxed and the molecules in the bottom bilayer are frozen to mimic bulk ice. The periodic boundary condition along the $c$-axis is then removed to create a surface. The $\mathrm{CO}$ admolecule is then added on the surface and all free coordinates are relaxed again. With this method, we generated three samples with different proton ordering, which we refer to as samples 1,2 , and 3 .

The KMC trajectories give the position of the $\mathrm{CO}$ admolecule, $\boldsymbol{r}$, on the surface as a function of time. This allows us to extract the diffusion constants, $D$, using the EinsteinSmoluchowski equation for two-dimensional diffusion:

$$
D=\lim _{t \rightarrow \infty} \frac{1}{4 t}\left\langle|\boldsymbol{r}(0)-\boldsymbol{r}(t)|^{2}\right\rangle .
$$

\section{Results}

\subsection{Binding energies and process barriers}

The PESs of the three samples were explored by performing AKMC simulations at $T=50 \mathrm{~K}$. We found 100,97 and 97 states for samples 1, 2 and 3 respectively. The processes in the TOEs only involve significant translation and rotation of the $\mathrm{CO}$ admolecule. This is because the temperature is too low to allow for reorientations of water molecules in the substrate, for which energy barriers are at least $200 \mathrm{meV}$ (about $2300 \mathrm{~K})$. As a result, the states which are found remain limited in number and form a closed system at any temperature below $T=50 \mathrm{~K}$. By a closed system we mean a system with a TOE which, regardless of the initial state, does not enter a new state even after taking an arbitrarily large number of KMC steps. This allows for long KMC runs without having to perform additional saddle point searches, because no new states are discovered.

The binding sites on sample 2 are shown in the left panel of Fig. 4. Even though the oxygen atoms in the substrate form a crystal lattice, large differences in admolecule orientation between the unit cells in the substrate can be seen. These differences are due to the proton disorder in the ice. This already shows the importance of having an unbiased method to find minima on the PES.

Typically, the admolecule is either at the center of a hexagon, or on top of one of the water molecules in the lower half of the upper half bilayer of the sample. The orientation of the $\mathrm{CO}$ molecule is mainly determined by the configuration 

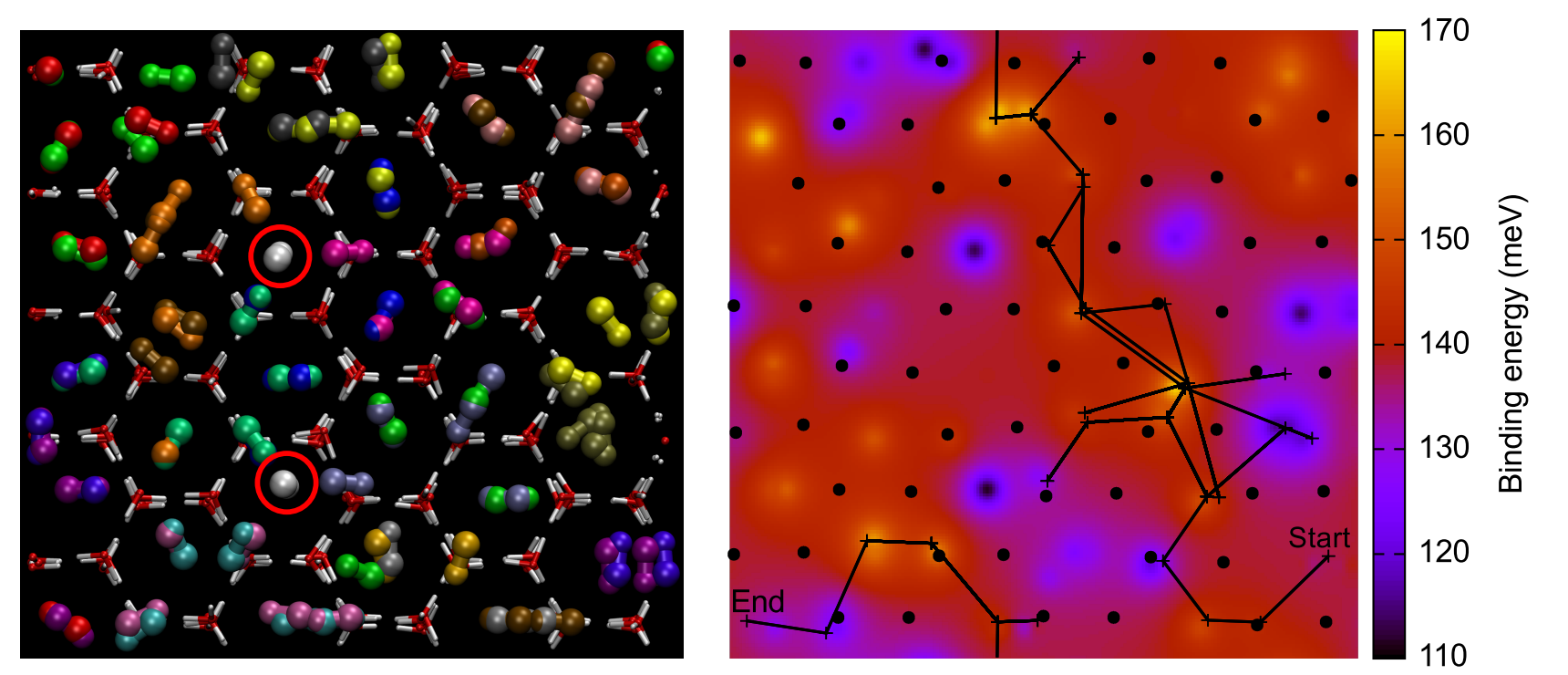

Fig. 4 Left panel) illustration of all the binding sites of the $\mathrm{CO}$ admolecule on ice $\mathrm{I}_{\mathrm{h}}$ substrate 2. Configurations of the same color belong to the same coarse grained composite state. The maximum size of these composite states was limited to 5 in this case. 95 Of the 97 states were coarse grained, the remaining two configurations are circled in red; the admolecule has a perpendicular orientation w.r.t. the surface in both cases. Right panel) binding energy of the $\mathrm{CO}$ admolecule on the surface of substrate 2. Only the energies of the minima have been included, the saddle points have been left out. The black dots denote the positions of the oxygen atoms in the upper half bilayer of the substrate. The black lines show part of a typical trajectory of the admolecule as it diffuses over the substrate by hopping from state to state. The start and end states of this trajectory are indicated in the figure.

of nearest neighbor dangling protons pointing out of the surface, which can be $0,1,2$ or 3 in number. The orientation is parallel to the surface if there is at least one nearest neighbor dangling proton. Without a dangling proton in the vicinity, the binding site at the center of a hexagon was found to yield a standing CO molecule, having its orientation parallel to the $c$ axis in the substrate. Two such configurations were found on sample 2 and have been marked by red circles in Fig. 4. These counterintuitive configurations again stress the importance of having an unbiased algorithm to explore the PES. A similar variety in binding has been observed in simulations of $\mathrm{H}_{2} \mathrm{O}$ admolecule diffusion on the ice $\mathrm{I}_{\mathrm{h}}(0001)$ surface ${ }^{46}$.

Another noteworthy feature of Fig. 4 is that there are many pairs of states in which the position of the $\mathrm{CO}$ molecule is almost identical, but its orientation is different by about 180 degrees. These states are connected to each other by processes which flip the molecule. These processes have relatively high energy barriers ( $60 \mathrm{meV}$ on average) while the configurational energies of the states differ on average by only $5 \mathrm{meV}$.

Besides the admolecule orientation, also the binding energies are strongly influenced by the proton disorder in the substrate, as can be seen in the right panel of Fig. 4. This figure shows the binding energy of the $\mathrm{CO}$ molecule in the states on the surface of sample 2, interpolated to a color map. The anisotropy of the PES is a clear demonstration of the large in- fluence of proton disorder on the binding energy. Naturally, the energies of the saddle points in between the binding sites, and the corresponding energy barriers, also show a large variation. This appears in the histogram of energy barriers in Fig. 5. Energy barriers for $\mathrm{CO}$ diffusion are roughly found to be between 0 and $75 \mathrm{meV}$, with a few processes which have higher barriers. The time-averaged binding energy, averaged over KMC runs on each of the three substrates at $T=50 \mathrm{~K}$ is $154 \pm 4 \mathrm{meV}$. This value is higher than the experimental value of $115 \mathrm{meV}$ for the adsorption energy of $\mathrm{CO}$ at submonolayer coverage on crystalline water ice, which was determined by temperature programmed desorption ${ }^{47}$. A possible explanation for this discrepancy is that the model charges on the $\mathrm{H}_{2} \mathrm{O}$ molecules do not change with the internal geometry of the molecule. With the CCL intramolecular potential, this leads to an increased dipole moment of the water molecules which, in turn, leads to stronger binding energies of the $\mathrm{CO}$ admolecule. An additional effect may be that the dipole moments of the water molecules at the surface can vary significantly depending on the local arrangement, as was observed in recent DFT calculations ${ }^{48}$. Because the Tip4p/Ice potential was designed to reproduce bulk properties, the resulting effect on the binding energy is not taken into account. Both effects however, are present at both the potential energy minima and the saddle points, 


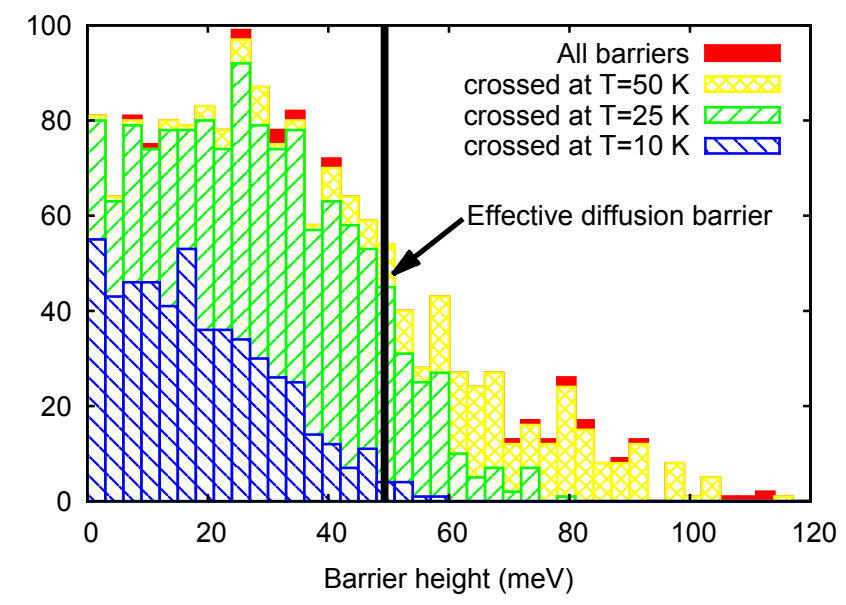

Fig. 5 Distribution of process energy barriers for $\mathrm{CO}$ diffusion on hexagonal ice. The highest, red, bars represent all processes in the table of events, irrespective of whether or not the barrier is ever crossed during a KMC run. The other bars show the barriers which are crossed during a KMC simulation at the specified temperature. The vertical black line shows the effective diffusion barrier of 49.4 $\mathrm{meV}$. Energy barriers from all three substrates were included in the data for this figure.

the height of the process barriers, and thereby the diffusion rate, remains unaffected. In summary, we expect the largest error to be on the desorption energy due to the use of a simple bulk potential and only to a lesser extent to the diffusion barriers. The calculated binding energies are significantly larger than the diffusion barriers. This implies that thermal desorption will occur on a much longer timescale than surface diffusion. Consequently, it is no problem that desorption processes are absent in our TOEs ${ }^{\S}$.

\subsection{Coarse graining}

A common problem in KMC simulations occurs when transition rates in the TOE vary significantly. In this situation, the simulation may become stuck, crossing low barriers over and over again. To avoid spending CPU cycles on simulating this uninteresting behavior, a coarse graining (CG) algorithm ${ }^{49}$ can be applied, which merges states connected by low barriers into composite states.

In our systems, the broad distribution of the energy barriers (see Fig. 5) leads to transition rates which vary over many or-

$\S$ It turns out that the minimum mode following method does not let the admolecule climb up from the surface during the saddle point searches. Even though it is, in principle, possible to find a saddle point which lets the admolecule desorb, it is unlikely that the system will relax back from the transition state to the exact reactant state where the saddle point search started (which is required for a successful saddle point search). It should be noted though that it is straightforward to include desorption in the TOEs by hand, should one want to.

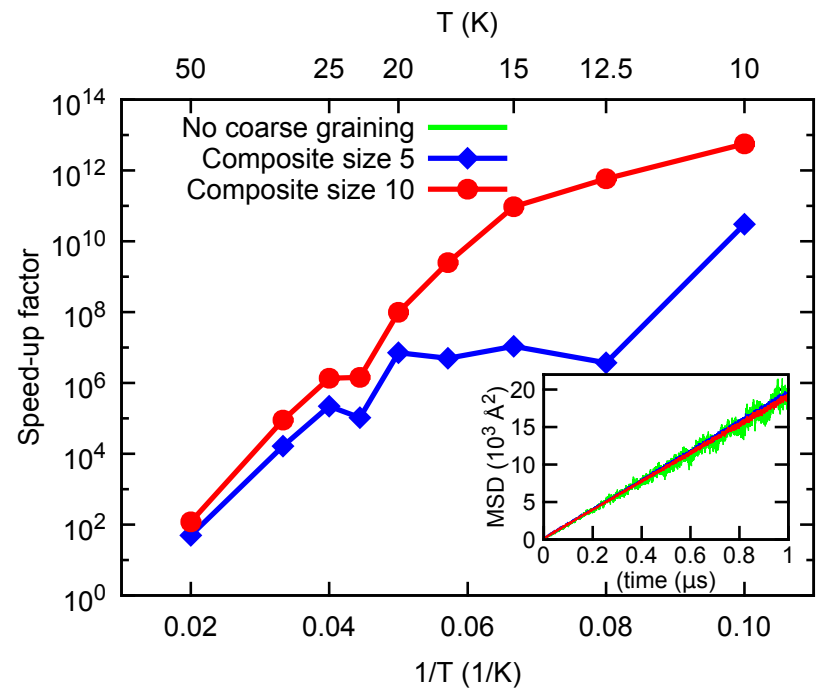

Fig. 6 Speed-up of KMC simulations obtained through coarse graining as a function of temperature. Speed-up is defined as the factor by which the simulated time increases with respect to a simulation without coarse graining. The inset shows the mean squared displacement (MSD) of the $\mathrm{CO}$ admolecule determined from a $2 \times 10^{7}$ iteration KMC simulation with and without coarse graining.

ders of magnitude, especially at low temperatures. This was the motivation for applying the CG algorithm, which is implemented in the EON code. For our purpose, however, we used the algorithm in a slightly modified form. Since we are interested in the position of the admolecule as the simulation progresses, we cannot allow the composite states to become arbitrarily large since this would eventually remove all spatial resolution. We therefore impose a limit on the maximum number of states inside a composite state. To ensure that the application of the CG algorithm does not change the physical properties of the system we will show later that the behavior of the mean squared displacement, as a function of time is the same with and without coarse graining.

To analyze the efficiency of the CG algorithm we calculate the speed-up factor. This factor is shown in Fig. 6, as a function of temperature and for different maximum size of the composite states. The speed-up is defined as the factor by which the simulated time in a KMC run increases with respect to a simulation without the $\mathrm{CG}$ algorithm. It naturally depends on the temperature and on the maximum allowed size of the composite states. Temperature determines the rates in the TOE through equation (5) and thereby also the relative probability of a process being picked by the KMC routine. The maximum size of the composite states effectively limits the lowest energy barrier in the system, which, in turn, determines the state with the lowest lifetime. At lower tempera- 
tures, the effect of the $\mathrm{CG}$ algorithm becomes quite dramatic indeed. For example, at $10 \mathrm{~K}$, a KMC simulation on sample 3 had simulated $1.2 \times 10^{9} \mathrm{~s}$ and visited 15 of the 97 states after $2 \times 10^{7}$ iterations. With the coarse graining algorithm applied, the simulated time increased by eight orders of magnitude to $2.3 \times 10^{17} \mathrm{~s}$ (comparable to the lifetime of the universe), after which 92 states were visited. Another factor which affects the speed-up is the way in which the states are merged into composite states. Depending on the initial random seed of the simulation, different states will be grouped together and some arrangements are more efficient than others. As an example, the color coding in Fig. 4 shows a particular choice of composite states on substrate 2 .

The time scales reached with the $\mathrm{CG}$ algorithm are huge at low temperatures, even for an interstellar molecular cloud. However, the diffusion constant extracted from the admolecule trajectory is still realistic. As is shown in the inset of Fig. 6, the mean squared displacement of the admolecule as a function of time does indeed not change when the CG algorithm is applied.

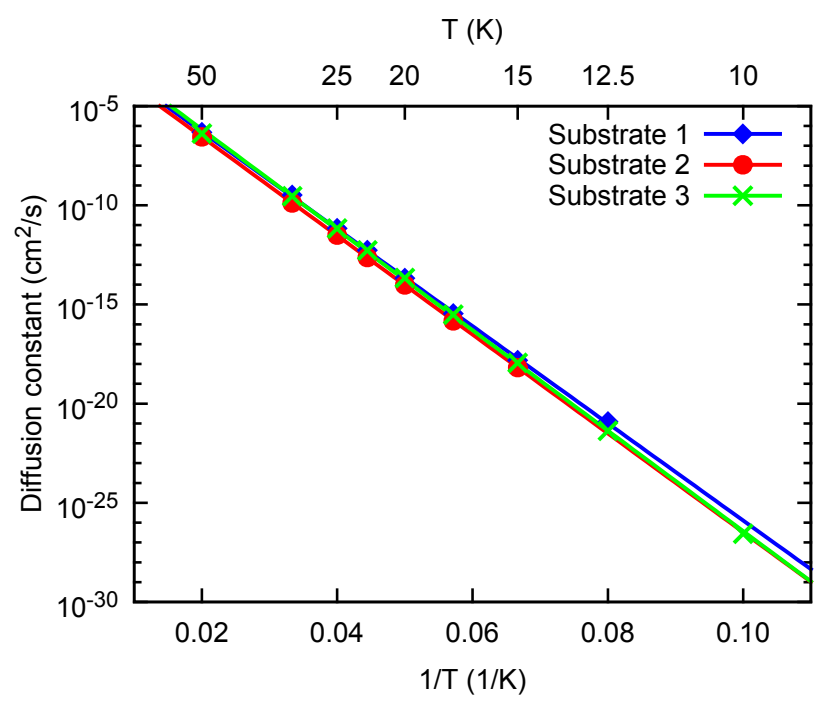

Fig. 7 Arrhenius plot of the diffusion constants of the $\mathrm{CO}$ admolecule on the surface of the three substrates. The lines show the best fit of an Arrhenius type function through the data points. The nearly equivalent slopes of the curve show that the effective diffusion barrier is similar on all three samples.

\subsection{Diffusion constants}

The surface diffusion constants of the $\mathrm{CO}$ admolecule were calculated from the mean squared displacements in the KMC trajectories at different temperatures using Eq. (6). In all simulations, the CG algorithm was applied with a maximum composite state size of 10 . At temperatures of $15 \mathrm{~K}$ and higher, the admolecule trajectories were found to be sufficiently long to extract a diffusion constant for all three samples. Despite the $\mathrm{CG}$ algorithm, suitable trajectories at $12.5 \mathrm{~K}$ could only be obtained on substrates 1 and 3, and at $10 \mathrm{~K}$ only for substrate 3. From this we can infer that long, surface-crossing paths, which are accessible at low temperatures, are found most easily on sample 3, whereas these kind of paths are harder to find on samples 1 and 2 .

The resulting diffusion constants are shown in Fig. 7. An Arrhenius type behavior is observed, with only slight differences between the three samples. The corresponding energy barriers and pre-exponential factors were extracted from this data and are listed in Table 2. The activation energy for diffusion is similar for the three samples. It can be interpreted as an effective diffusion barrier on the substrate, i.e., it is the highest barrier the admolecule has to cross along the minimum energy path across the surface. The average effective energy barrier is also shown in Fig. 5, together with the energy barriers which are crossed at 50,25 and $10 \mathrm{~K}$. The effective barrier is just at the end of the range of barriers which are still accessible at $T=10 \mathrm{~K}$. The variation in the pre-exponential factor, $D_{0}$, is larger. On a perfectly periodic surface, this quantity depends on the process prefactor, the number of processes from each state and the hopping distance. In our case, the differences are likely related to the pattern of dangling protons on the surface. Since the dangling protons largely determine the binding energies and height of process barriers they dictate which lowenergy paths the admolecule can take across the surface. As we argued earlier, such paths are most easily found on substrate 3 . It is therefore not surprising that the pre-exponential factor is largest in this sample, since the admolecule can move about most easily.

The calculations presented here have assumed the atoms can be described as classical particles. At low temperatures however, tunneling will become the dominant transition mechanism. The AKMC simulation approach can include tunneling as a possible mechanism. From the imaginary frequency of the unstable mode at each saddle point, an estimate of the crossover temperature, $T_{c}$, for tunneling can be estimated ${ }^{50}$, and if the temperature of interest is lower than this value, the saddle point search can be refined to include quantum mechanical effects (to converge on so-called instantons). The transition rate is then evaluated from a harmonic quantum TST

Table 2 Effective diffusion energy barrier and pre-exponential factor $D_{0}$ for CO diffusion on the three difference ice $\mathrm{I}_{\mathrm{h}}$ substrates

\begin{tabular}{lll}
\hline Substrate & Barrier $(\mathrm{meV})$ & $D_{0}\left(\mathrm{~cm}^{2} \mathrm{~s}^{-1}\right)$ \\
\hline 1 & $48.6 \pm 0.6$ & $(4.08 \pm 1.36) \times 10^{-2}$ \\
2 & $49.5 \pm 0.2$ & $(2.76 \pm 0.42) \times 10^{-2}$ \\
3 & $50.3 \pm 0.6$ & $(7.94 \pm 1.80) \times 10^{-2}$ \\
\hline
\end{tabular}


rather than a classical harmonic $\mathrm{TST}^{51,52}$. In our simulations the average $T_{c}$ was found to be $6 \pm 5 \mathrm{~K}$, which justifies the use of the classical approximation.

\section{Conclusions}

The modeling of interstellar ices still poses a formidable challenge for modern-day simulation techniques. Kinetic Monte Carlo techniques are a powerful tool to study these complex systems over long time spans. In this paper we proposed a particular approach which combines both on- and offlattice KMC simulations. Whereas off-lattice KMC simulations have demonstrated their use before, off-lattice adaptive Kinetic Monte Carlo has not been used for the simulation of molecular systems before. We have demonstrated the potential power of AKMC simulations by performing calculations on a system of a $\mathrm{CO}$ admolecule on an hexagonal ice surface. Despite the presence of strong intramolecular forces, the minimum-mode following algorithm was able to locate saddle points on the PES of the system efficiently to populate the TOE needed for the KMC simulation.

Due to the proton disorder in the ice substrate, the binding sites of the admolecule were found to show a large variation in both energy and configuration. This shows that, even for this relatively simple system, it is hard to construct a TOE without having an unbiased search algorithm. From the TOE we could extract information such as the average binding energy and the height and spread of diffusion energy barriers. Also, typical processes which can occur in the system, like a flip of the $\mathrm{CO}$ molecule, could be characterized. These are all examples of the kind information one would need beforehand when constructing a TOE for performing on-lattice KMC simulation of the same system. This illustrates why the combination of off-lattice AKMC and on-lattice KMC is a potentially very useful simulation technique. When combining the two techniques however, one has to be careful when translating the detailed AKMC data into a lattice model. For nearly crystalline system, as presented in this paper, one should make sure the lattice structure represents the crystal structure as much as possible. In this case one would ideally use an hexagonal lattice structure with two sites per lattice point to reproduce the binding sites at the center of a hexagon and those on top of a water molecule. When dealing with amorphous systems, one should choose a lattice which reproduces the average number of nearest neighbors correctly and make sure that the processes and their rates are dependent on the environment (number, kind and possibly orientation of neighbors).

Through the application of a coarse graining algorithm we have shown that the timescales accessible by AKMC can be greatly enhanced without changing the physical properties of the system. This is especially important at low temperatures, where the interesting dynamics takes places on the longest timescales. From the KMC trajectories we have extracted the diffusion coefficient for $\mathrm{CO}$ on hexagonal ice. From the temperature dependence of the diffusion coefficients, the effective diffusion barrier was determined to be $50 \pm 1 \mathrm{meV}$. The effect of proton disorder of the ice substrate becomes particularly noticeable at temperatures below $15 \mathrm{~K}$; in this situation the admolecule can get trapped in certain regions of the surface making large scale movement of the admolecule impossible.

The main conclusion from this work is that we have shown that AKMC is a promising technique to study kinetic processes in complex molecular systems like interstellar ices. Its unbiased saddle point searches, combined with realistic potential energy functions, allow for the identification of transition mechanisms which one cannot guess beforehand based on intuition alone. This unbiased approach is really the power of AKMC since it gives detailed chemical insight about which kinetic processes are crucial in the dynamical evolution of the ices. However, for the simulation of bulk processes in amorphous ices, the AKMC method is still too expensive to cover astronomically relevant timescales of large systems. We therefore believe that using the detailed chemical knowledge, obtainable from AKMC, as input for coarser on-lattice KMC simulations will be a major step forward in understanding the structure and dynamics of interstellar ices.

\section{Acknowledgements}

This work has been funded by the European Research Council (ERC-2010-StG, Grant Agreement no. 259510-KISMOL), COST Action Number CM0805 (The Chemical Cosmos: Understanding Chemistry in Astronomical Environments) and the Icelandic Research Fund administrated by RANNIS. We gratefully acknowledge M.C. van Hemert for fruitful discussions and for providing the CO potential and J.C. Berthet for help with the implementation of the water potential in EON.

\section{References}

1 K. I. Öberg, E. C. Fayolle, H. M. Cuppen, E. F. van Dishoeck and H. Linnartz, Astron. Astrophys., 2009, 505, 183.

2 J. T. H. van Eupen, W. W. J. Elffrink, R. Keltjens, P. Bennema, R. de Gelder, J. M. M. Smits, E. R. H. van Eck, A. P. M. Kentgens, M. A. Deij, H. Meekes and E. Vlieg, Cryst. Growth Des., 2008, 8, 71.

3 J. Los, W. J. P. van Enckevort, H. Meekes and E. Vlieg, J. Phys. Chem. B, 2007, 111, 782 .

4 A. M. Nienow and J. T. Roberts, Annu. Rev. Phys. Chem., 2006, 57, 105.

5 K. Muralidharan, P. Deymier, M. Stimpfl, N. H. de Leeuw and M. J. Drake, Icarus, 2008, 198, 400.

6 D. Bockelée-Morvan, D. C. Lis, J. E. Wink et al., Astron. Astrophys., 2000, 353, 1101

7 S. Viti, M. P. Collings, J. W. Dever, M. R. S. McCoustra and D. A. Williams, Mon. Not. R. Astron. Soc., 2004, 354, 1141. 
8 R. T. Garrod, V. Wakelam and E. Herbst, Astron. Astrophys., 2007, 467, 1103.

9 E. Herbst and E. F. van Dishoeck, Annu. Rev. Astron. Astr., 2009, 47, 427.

10 E. L. Gibb, D. C. B. Whittet, A. C. A. Boogert and A. G. G. M. Tielens, Astrophys. J. Suppl. S., 2004, 151, 35.

11 A. C. A. Boogert, K. M. Pontoppidan, C. Knez et al., Astrophys. J., 2008, 678, 985.

12 H. M. Cuppen, E. M. Penteado, K. Isokoski, N. van der Marel and H. Linnartz, Mon. Not. R. Astron. Soc., 2011, 417, 2809.

13 K. M. Pontoppidan, A. C. A. Boogert, H. J. Fraser, E. F. van Dishoeck, G. A. Blake, F. Lahuis, K. I. Öberg, N. J. Evans, II and C. Salyk, Astrophys. J., 2008, 678, 1005.

14 P. Caselli, T. I. Hasegawa and E. Herbst, Astrophys. J., 1993, 408, 548.

15 R. T. Garrod, S. L. W. Weaver and E. Herbst, Astrophys. J., 2008, 682, 283.

16 A. I. Vasyunin, D. A. Semenov, D. S. Wiebe and T. Henning, Astrophys. J., 2009, 691, 1459.

17 M. P. Collings, M. A. Anderson, R. Chen, J. W. Dever, S. Viti, D. A. Williams and M. R. S. McCoustra, Mon. Not. R. Astron. Soc., 2004, 354, 1133.

18 H. M. Cuppen, E. F. van Dishoeck, E. Herbst and A. G. G. M. Tielens, Astron. Astrophys., 2009, 508, 275.

19 A. G. G. M. Tielens and W. Hagen, Astron. Astrophys., 1982, 114, 245.

20 T. I. Hasegawa, E. Herbst and C. M. Leung, Astrophys. J. Suppl. S., 1992, 82, 167.

21 P. Caselli, T. I. Hasegawa and E. Herbst, Astrophys. J., 1998, 495, 309.

22 S. Ioppolo, H. M. Cuppen, C. Romanzin, E. F. van Dishoeck and H. Linnartz, Astrophys. J., 2008, 686, 1474.

23 G. W. Fuchs, H. M. Cuppen, S. Ioppolo, C. Romanzin, S. E. Bisschop, S. Andersson, E. F. van Dishoeck and H. Linnartz, Astron. Astrophys., 2009, 505, 629.

24 B. Barzel and O. Biham, Astrophys. J. Lett., 2007, 658, 37.

25 H. M. Cuppen and E. Herbst, Astrophys. J., 2007, 668, 294.

26 S. X. M. Boerrigter, G. P. H. Josten, J. van de Streek, F. F. A. Hollander, J. Los, H. M. Cuppen, P. Bennema and H. Meekes, J. Phys. Chem. A, 2004, 108, 5894-5902.

27 G. Henkelman and H. Jónsson, J. Chem. Phys., 2001, 115, 9657.

28 A. Pedersen and H. Jónsson, Math. Comput. Simulat., 2010, 80, 1487.

29 H. Jónsson, Proc. Natl. Acad. Sci. USA, 2011, 108, 944.

30 G. Henkelman and H. Jónsson, J. Chem. Phys., 1999, 111, 7010.

31 http://www. theochem.org/EON.

32 H. Vineyard, J. Phys. Chem. Solids, 1957, 3, 121.

33 K. I. Öberg, E. C. Fayolle, H. M. Cuppen, E. F. van Dishoeck and H. Linnartz, Astron. Astrophys., 2009, 505, 183.

34 S. Cazaux, V. Cobut, M. Marseille, M. Spaans and P. Caselli, Astron. Astrophys., 2010, 522, A74.

35 A. Pedersen and H. Jónsson, Acta Material., 2009, 57, 4036.

36 A. Pedersen, G. Henkelman, J. Schiøtz and H. Jónsson, New J. Phys., 2009, 11, 073034.

37 J. L. F. Abascal, E. Sanz, R. García Fernández and C. Vega, J. Chem. Phys., 2005, 122, 234511.

38 S. K. Langhoff, L. A. Curtiss and G. Carney, J. Mol. Spectrosc., 1976, 61, 371.

39 M. C. van Hemert, Private Communication, 2011.

40 A. Mantz and J. Maillard, J. Mol. Spectrosc., 1975, 57, 155.

41 V. I. Vedeneyev, L. V. Gurvich, V. N. Kondratyev, V. A. Medvedev and Y. L. Frankevich, Bond Energies, Ionization Potentials and Electron Affinities, St. Martin's Press, New York, 1962.

42 P. Bunker, J. Mol. Spectrosc., 1972, 5, 478.

43 A. Pedersen, S. F. Hafstein and H. Jónsson, Siam. J. Sci. Comput., 2011, 33, 633.

44 R. Malek and N. Mousseau, Phys. Rev. E, 2000, 62, 7723.
45 V. Buch, P. Sandler and J. Sadlej, J. Phys. Chem. B, 1998, 102, 8641.

46 E. Batista and H. Jónsson, Comp. Mater. Sci., 2001, 20, 325.

47 J. A. Noble, E. Congiu, F. Dulieu and H. J. Fraser, Mon. Not. R. Astron. Soc., 2012, 421, 768.

48 M. Watkins, D. Pan, E. G. Wang, a. Michaelides, J. VandeVondele and B. Slater, Nat. Mat., 2011, 10, 794

49 A. Pedersen, J. C. Berthet and H. Jónsson, Lect. Notes. Comput. Sc., 2012, 7134, 34.

50 M. Gillan, J. Phys. C Solid State, 1987, 20, 3621.

51 G. Mills, G. K. Schenter, D. E. Makarov and H. Jónsson, Chem. Phys. Lett., 1997, 278, 91.

52 S. Andersson, G. Nyman, A. Arnaldsson, U. Manthe and H. Jónsson, J. Phys. Chem. A, 2009, 113, 4468.

10 | Journal Name, 2010, [vol], 1-10 